\title{
The aperiodic nature of incommensurately modulated structures ${ }^{\star}$
}

\author{
Gervais Chapuis · Alla Arakcheeva
}

Received: date / Accepted: date

\begin{abstract}
The discovery of aperiodic crystals is perhaps one of the most important event which has changed our vision on crystalline architectures since the discovery of diffraction hundred years ago. It was the merit of a dutch crystallographer, P.M. de Wolff, to interpret their diffraction pattern as a three dimensional projection of a higher dimensional reciprocal lattice, idea which led directly to the generalization of the concept of crystal. Aperiodic crystals are currently described as periodic objects in higher-dimensional space, i.e. the superspace and their structures can be described in terms of 3-d cuts. Incommensurate structures, composite structures and quasicrystals all belong to aperiodic structures.

Many interesting properties of superspace have been discovered which are also directly applicable to crystals in the conventional sense, i.e. crystals with 3 -d periodicity. In particular the concept of structure type can be extended for a better understanding of structure relations. The notion of solid solution has also benefited from superspace considerations. Moreover, superspace is a very powerful tool for a better understanding of structure-property relations in material science, e.g. luminescence properties could be directly associated to the description of structures in superspace. Recently, this concept has been used for the prediction of new structural modifications including polytypes and even polytypic modifications of a well known pharmaceutical product.
\end{abstract}

G. Chapuis

Laboratoire de cristallographie, BSP/Cubotron, EPFL, 1015 Lausanne, Switzerland

Tel.: +41-21-6930630

Fax: +41-21-6930634

E-mail: gervais.chapuis@epfl.ch

A. Arakcheeva

Phase Solutions Ltd, ch. des Mésanges 7, 1012 Lausanne, Switzerland

1 This contribution is the written, peer-reviewed version of a paper presented at the conference "The Centennial of X-Ray Diffraction (1912-2012)", held at Accademia Nazionale dei Lincei in Rome on May 8 and 9, 2012. 
Keywords Aperiodic crystals - Incommensurately modulated crystals · Composite crystals · Quasicrystals

PACS 61.44.Fw $\cdot$ 61.44.Br $\cdot$ 77.84.Lf $\cdot 61.50 . \mathrm{Ah}$

\section{Introduction and historical summary}

The celebration of the hundredth anniversary of the discovery of diffraction by crystalline solids (1912-2012) gives us an excellent opportunity to look at the impressive contribution of diffraction methods towards our knowledge on the structure of matter. The enormous progress is due to theoretical and experimental advances and today, even the finest structural details of large objects like ribosomes are accessible by diffraction methods.

The three dimensional periodicity of crystalline structures is a fundamental concept which is at the basis of the theoretical model which has been so successful for diffraction. However, already in the late 1920's, the three dimensional concept of lattice periodicity was already put into doubt on the basis of diffraction diagrams of laminated metals. Dehlinger [1] explained the line broadening of Debye-Scherrer diagrams by periodic perturbation and invented the name of Gittergeister to characterize the additional lines appearing on the diagrams. Preston [2] later introduced the term satellites to characterize the additional lines observed on aluminium copper alloys. Shortly after, Kochendörfer [3] calculated the intensities of Debye-Scherrer lines resulting from a sinusoidal wave of atomic displacements. Daniel and Lipson [4] further developed the theory of periodic perturbation on the basis of $\mathrm{Co}-\mathrm{Ni}-\mathrm{Fe}$ alloy diffractograms. They were however not able to conclude on the nature of the perturbation, was it a scattering modulation or a lattice modulation? Hargreaves [5] extended their work by considering rectangular modulations in $\mathrm{Cu}-\mathrm{Ni}-\mathrm{Fe}$ and postulated that

the magnetic properties of the alloys are systematically affected by the development of the modulated structure.

The existence of satellite reflections was not only observed in metal alloys but also in felspars [6].

The most significant advance in the theory of periodic distortions in crystalline structures is due to Korekawa [7]. In his habilitation thesis, he presented a complete series of possible distortions by combining transversal or longitudinal displacement waves along with density waves and estimated the intensities of the satellite reflections consequently to the amplitude of the periodic perturbations. His theoretical work was illustrated with two real cases, namely the structures of the mineral labradorite and an organic copper containing complex. This work was published in German and not easily accessible and consequently its diffusion was limited. Another limitation was due to the lack of symmetry considerations regarding the theory of satellites.

The real breakthrough came later in 1972 when de Wolff [8] and Janner [9] presented independently their work in the IUCr conference in Kyoto on the 
four-dimensional space group of $\gamma \mathrm{Na}_{2} \mathrm{CO}_{3}$ respectively the symmetry groups of lattice vibrations. Both presenters realized that they were using symmetry groups with the same properties and thus the tools for the complete symmetry description of periodically distorted crystal structures were established. The introduction of an additional space dimension to describe the structure of $\gamma \mathrm{Na}_{2} \mathrm{CO}_{3}$ was justified by the existence of a periodic distortion, i.e. a modulation component which is independent of the classical 3-d lattice periodicity. The term incommensurate structure was later introduced to describe modulations with irrational wave vector components.

An additional kind of structural complexity was described when Makovicky and Hyde published their work on misfit layer structures [10]. They considered incommensurate structures in the form of alternating layers possessing two independent periodicities and introduced the term incommensurate composites. It could be shown later that the symmetry of composites could perfectly well be described with the same higher dimensional groups introduced by de Wolff and Janner. Perhaps the most spectacular discovery in the structural departure of purely 3-d periodicity appeared when Shechtman and coworkers published their work on a metallic phase with long-range order but no translational symmetry [11]. The diffractograms of this phase exhibited perfect icosahedral symmetry which is obviously incompatible with 3 -d periodicity. The term quasicrystal (QC) was then introduced to describe crystalline structures with similar diffraction patterns [12]. Here again, it was soon realized that additional dimensions were necessary to describe the structural and symmetrical properties of QC.

In this article we shall first present a common concept, the so called superspace to describe the structures and symmetries of aperiodic crystals, which include incommensurate and composite structures as well as QC. Some examples of aperiodic structures will be presented and finally, we shall present an application of the superspace formalism in the field of materials science.

\section{Aperiodic structures and the superspace concept}

Let us first consider a diffraction layer of an incommensurate crystal as represented in fig. 1. We observe that the reflections at the intersection of the grid lines are all accompanied with satellite reflections along parallel directions to the modulation vector $\mathbf{q}$. It would be an impossible task to find a smaller reciprocal cell which would allow the indexing of all the spots with only two integers, knowing that the magnitude of the modulation vector $\mathbf{q}$ might change with temperature or pressure. It is thus natural to select an additional integer $m$ and index each spot on the layer with three integers as in eq. (1). This is also justified by the fact that the diffracted intensities decrease with the magnitude of the integer $m$.

$$
\mathbf{H}=h_{1} \mathbf{a}_{1}^{*}+h_{2} \mathbf{a}_{2}^{*}+m \mathbf{q}
$$




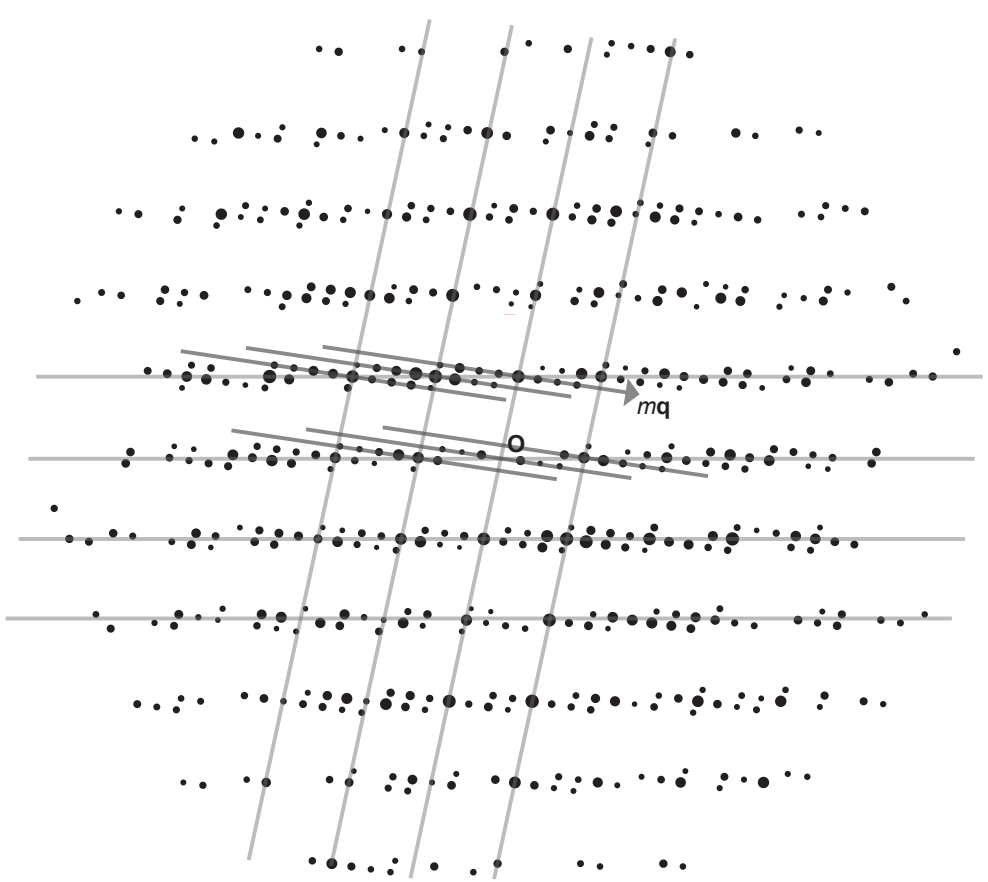

Fig. 1 Diffraction layer of an incommensurate crystal. Main reflections are located at the intersection of the grey lines whereas the satellite reflections are located along the darker lines at distances $m \mathbf{q}$ with integer $m$

the number of integers which are necessary to completely characterize a diffraction diagram are in the most general case given by eq. (2). The number of integers $n$ to fully characterize a diffraction diagram is called the rank and is different from the space dimension in which the corresponding structure is described.

$$
\mathbf{H}=h_{1} \mathbf{a}_{1}^{*}+h_{2} \mathbf{a}_{2}^{*}+h_{3} \mathbf{a}_{3}^{*}+\cdots+h_{n} \mathbf{a}_{n}^{*}
$$

We can now easily introduce the following definition:

Definition. $\mathbf{H}$ describes a periodic crystal if its space is equal to its rank. $\mathbf{H}$ describes an aperiodic crystal if its rank is larger than its space.

Aperiodic crystals have been observed in all types of crystalline materials including minerals, metals, alloys (quasicrystals), organic and inorganic materials, macromolecules. They also appear in pressure or temperature induced phase transitions $[13,14]$.

The introduction of additional integers for the characterization of aperiodic crystals is associated with additional space dimensions in reciprocal space. Fig. 


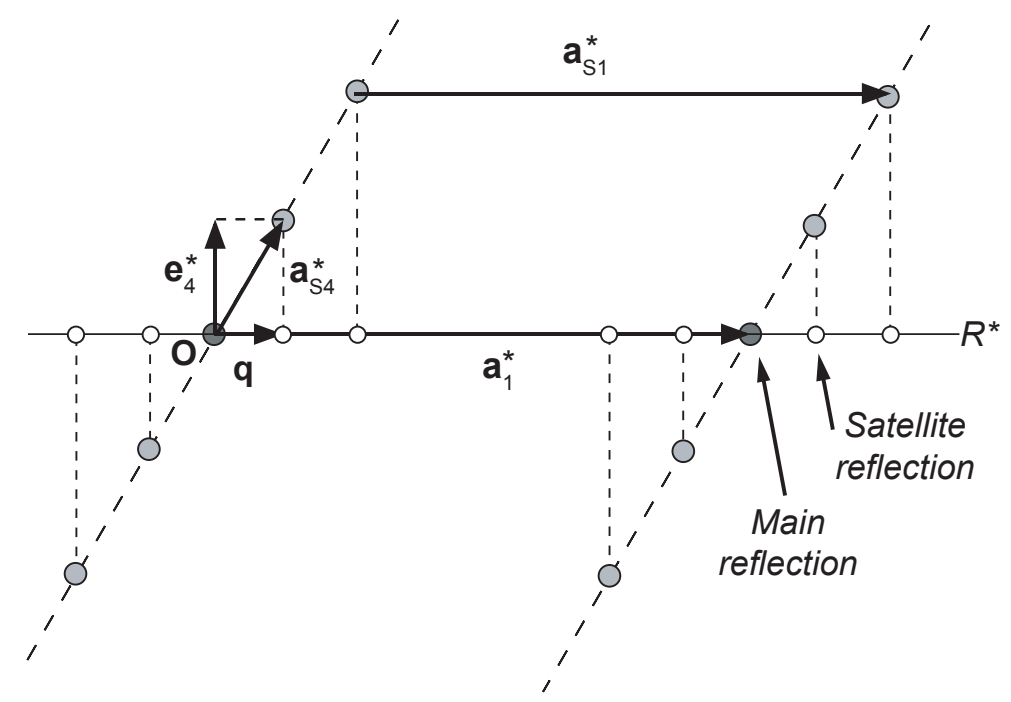

Fig. 2 Embedding of the reciprocal space in higher dimensional space, i.e. superspace. The satellite reflections along $\mathbf{a}_{1}^{*}$ are extended into two components, $\mathbf{e}_{4}^{*}$ and $\mathbf{q}$, to form the reciprocal lattice vector $\mathbf{a}_{S 4}^{*}$

2 illustrates the embedding of the reciprocal space in a space of higher dimension. We shall call this type of embedding superspace and use the subscript $S$ to characterize the corresponding reciprocal lattice vectors. For reasons which are beyond the scope of this presentation, the additional dimensions used to describe aperiodic crystals have some special properties. We therefore prefer to use the expression $(3+1) d$ to emphasize the additional dimension for the description of a three dimensional structure of rank 4 .

Given the basis vectors of an aperiodic crystal in reciprocal space, the next task is to find the corresponding vectors in direct space. For this purpose, we can use the scalar product relating direct and reciprocal basis vectors as in equation 3

$$
\left.\begin{array}{l}
\mathbf{a}_{S 1}^{*}=\left(\mathbf{a}_{1}^{*}, 0\right) \\
\mathbf{a}_{S 2}^{*}=\left(\mathbf{a}_{2}^{*}, 0\right) \\
\mathbf{a}_{S 3}^{*}=\left(\mathbf{a}_{3}^{*}, 0\right) \\
\mathbf{a}_{S 4}^{*}=(\mathbf{q}, 1)
\end{array}\right\} \rightarrow \mathbf{a}_{S i} \cdot \mathbf{a}_{S j}^{*}=\delta_{i j} \rightarrow\left\{\begin{array}{l}
\mathbf{a}_{S 1}=\left(\mathbf{a}_{1},-\mathbf{q} \cdot \mathbf{a}_{1}\right) \\
\mathbf{a}_{S 2}=\left(\mathbf{a}_{2},-\mathbf{q} \cdot \mathbf{a}_{2}\right) \\
\mathbf{a}_{S 3}=\left(\mathbf{a}_{3},-\mathbf{q} \cdot \mathbf{a}_{3}\right) \\
\mathbf{a}_{S 4}=(\mathbf{0}, 1)
\end{array}\right.
$$

$\delta_{i j}$ is the Kronecker symbol which is one if $i$ and $j$ are equal and zero otherwise. The direct space components of the lattice have two parts, the external respectively the internal components. In the former we recognize the conventional lattice vectors whereas in the latter, the scalar product of the modulation vector with the lattice vectors.

In order to get more insight into the superspace representation of aperiodic crystals, let us apply a periodic longitudinal displacement along a to a 

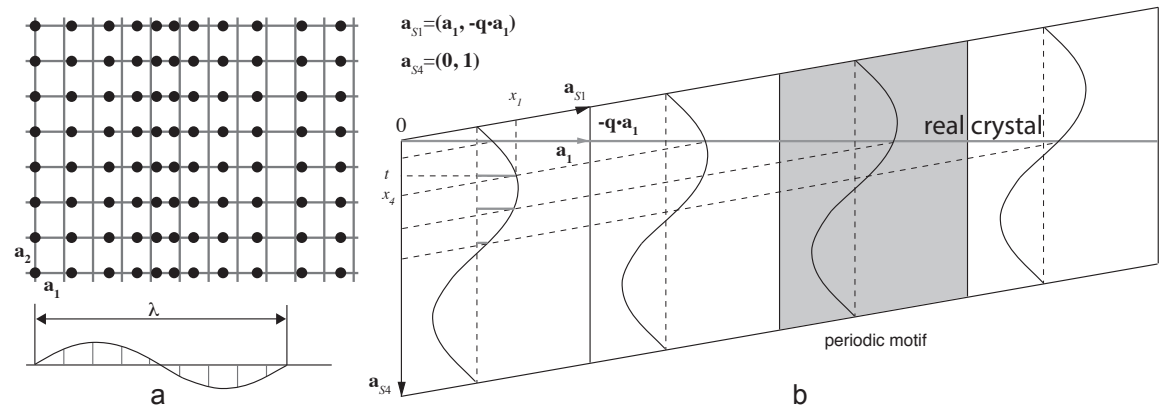

Fig. 3 a) Periodic longitudinal distortion with wavelength $\lambda$ applied to an array of grid points. b) Representation in superspace of the corresponding distortion in the section $\mathbf{a}_{S 1}, \mathbf{a}_{S 4}$.

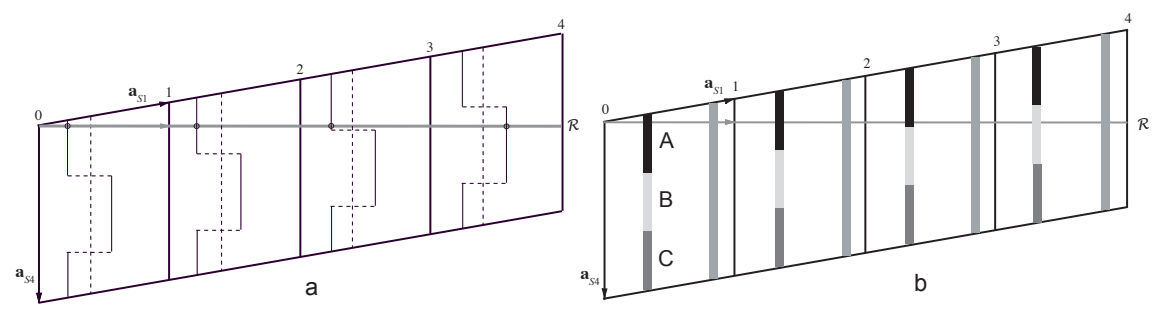

Fig. 4 Modulation functions can have many different shapes. a) Step function which might characterize atomic displacements over two sites. b) Example of a layer structure where one layer might choose either the $\mathrm{A}$ or $\mathrm{B}$ or $\mathrm{C}$ position.

periodic array as represented in fig. 3a. The modulation wave is parallel to $\mathbf{a}_{1}$ with wavelength $\lambda$ which is independent of the lattice periodicity. The corresponding representation of this modulation is illustrated in fig. $3 \mathrm{~b}$ for the section $\left(\mathbf{a}_{S 1}, \mathbf{a}_{S 4}\right)$. This two dimensional periodic array is given by the grey motif. The position of a single atom is given by the sinusoidal wave with a given amplitude. The components of the basis vectors in superspace according to relation (3) are indicated on the figure. The physical structure is given by the intersection of the periodic distortion with the horizontal line labelled "real crystal" which reproduces the deformation of fig. 3a. As the periodicity along $\mathbf{a}_{2}$ is not modulated, the corresponding representation of an atom in the section $\left(\mathbf{a}_{S 2}, \mathbf{a}_{S 4}\right)$ is obviously a straight line.

Since the introduction of superspace, many different types of modulation functions in addition to the harmonic modulation represented in fig. 3 have been introduced in order to improve the description of aperiodic crystals. Fig. 4 for example gives two functions which are frequently used. Fig. 4a characterizes a displacement of an atom onto two possible positions whereas fig. 4b illustrates layer displacements between the three possible layers positions A, $\mathrm{B}$ or $\mathrm{C}$. This list is not exhaustive and additional examples can be found e.g. in $[13]$ 

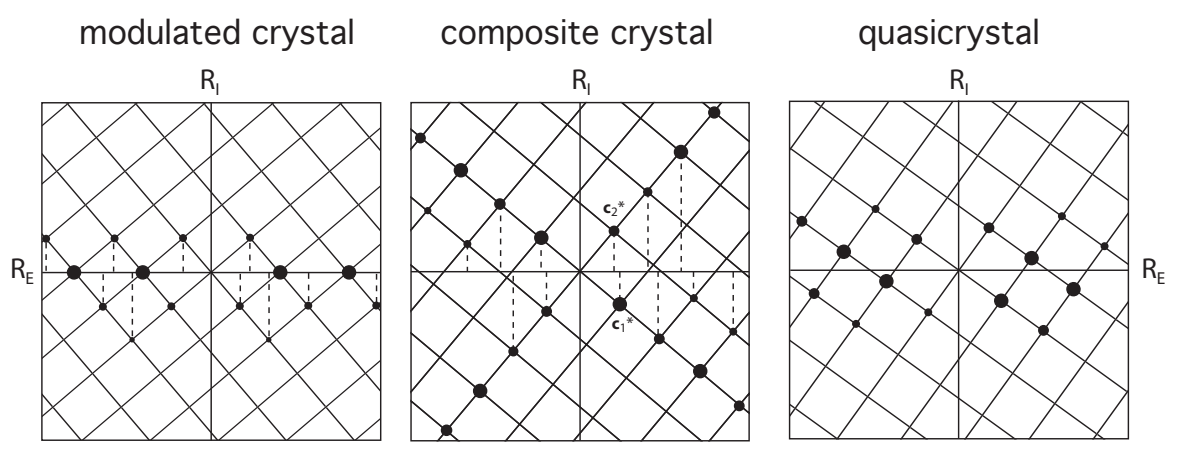

Fig. 5 Schematic representation of the reciprocal space indicating the essential differences between incommensurate, composite and quasi-crystals according to Yamamoto [16]. Note the positions of the reflections relative to the external and internal components of reciprocal space for each type of aperiodic structure.

The treatment of symmetry of aperiodic crystals is beyond the scope of this article. We can just mention that the resolution of aperiodic structures follows the same scheme as for conventional structures. The list of all possible superspace groups of e.g. $(3+1) \mathrm{d}$ has been established along with their corresponding systematic absences (see for example [15]). It is the task of the structure specialist to delimit the right superspace group and the corresponding modulation functions that best fit the observed intensities of the aperiodic structure under considerations.

Before ending this section, we would like to give some precisions about the various types of aperiodic crystals which we introduced in section 1 . What are the essential differences between incommensurate, composite and quasicrystals. Fig. 5 is inspired from the publication of Yamamoto [16] and uses the decomposition in external and internal components of reciprocal space to illustrate their main characteristics. Fig. 5a is nothing else than the extension already presented in fig. 2 for a modulated crystal. In this case, only the main reflections are contained along the external space dimension whereas the satellite reflections have both external and internal components. Composite crystals show essentially two main directions indicated by $c_{1}^{*}$ and $c_{2}^{*}$ as in fig. $5 \mathrm{~b}$. Satellite reflections are located on the grid points formed by these two directions but close to them. Finally, the representation for quasicrystals given in fig. $5 \mathrm{c}$ indicates that the reflections are located at the intersection of a grid, independently of the external and internal components.

\section{Examples of incommensurate structures}

For space limitations, we shall limit ourselves to a small selection of aperiodic structures namely incommensurate structures. Our first example is the structure of $\gamma-\mathrm{Na}_{2} \mathrm{CO}_{3}$ which is incommensurate over a large temperature do- 


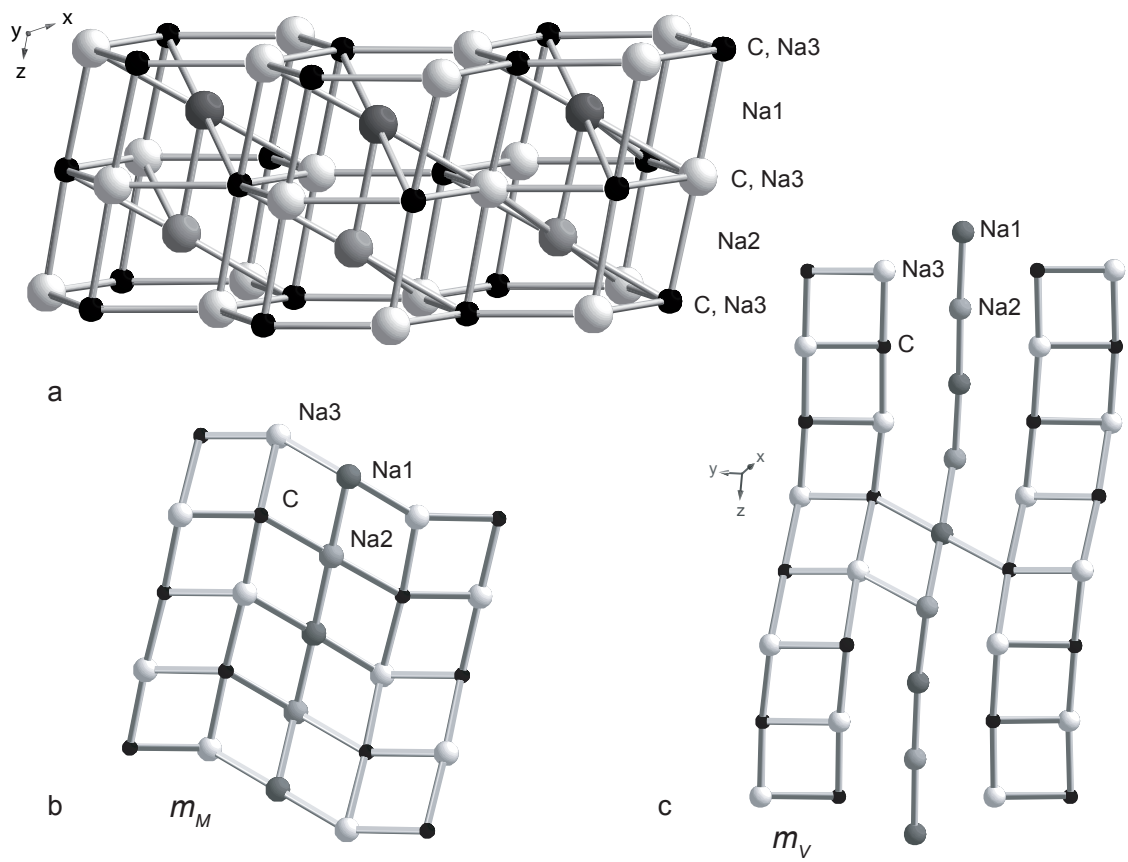

Fig. 6 a) Schematic representation of the incommensurate $\gamma \mathrm{Na}_{2} \mathrm{CO}_{3}$ in terms of graphite like layers. $\mathrm{O}$ atoms are omitted. b) Section of the incommensurate structure lying on the mirror plane $\mathrm{m}_{M}$. c) Section of the same structure along a different direction. In all three representations, we observe the unique role of $\mathrm{Na} 3$.

main, including room temperature. This is the first structure which was solved in the superspace formalism by de Wolff and his team [17]. The structure is represented in fig. 6a. For our purpose, we shall neglect the $\mathrm{O}$ atoms forming triangles around the $\mathrm{C}$ atoms. Thus, the structure consists of graphite like layers composed of $\mathrm{C}$ and $\mathrm{Na} 3$ stacked in the third dimension and in between, we find the Na1 and Na2 atoms. From the chemical point of view, it is interesting to note that $\mathrm{Na} 3$ has a completely different chemical environment than the two other $\mathrm{Na}$ atoms and can thus be reinterpreted as a different kind of atom for the structure characterization. At higher temperature, $\mathrm{Na}_{2} \mathrm{CO}_{3}$ is hexagonal with the hexagonal axis along c. At lower temperature, a monoclinic distortion occurs along b. Fig. $6 \mathrm{~b}$ shows a $\left(\mathbf{a}_{S 1}, \mathbf{a}_{S 3}\right)$ section where all atoms lie on the mirror plane $\mathrm{m}_{M}$. The monoclinic deformation can occur along three equivalent directions in the high temperature phase and only one is realized. The other two directions $\mathrm{m}_{V}$ are frustrated and attempt to establish a similar equilibrium environment as in the $\mathrm{m}_{M}$. This can only be accomplished by the introduction of a modulation which is illustrated in fig. $6 \mathrm{c}$. We can see that the environment found in $\mathrm{m}_{M}$ is only very partially realized in the $\mathrm{m}_{V}$ direction. 

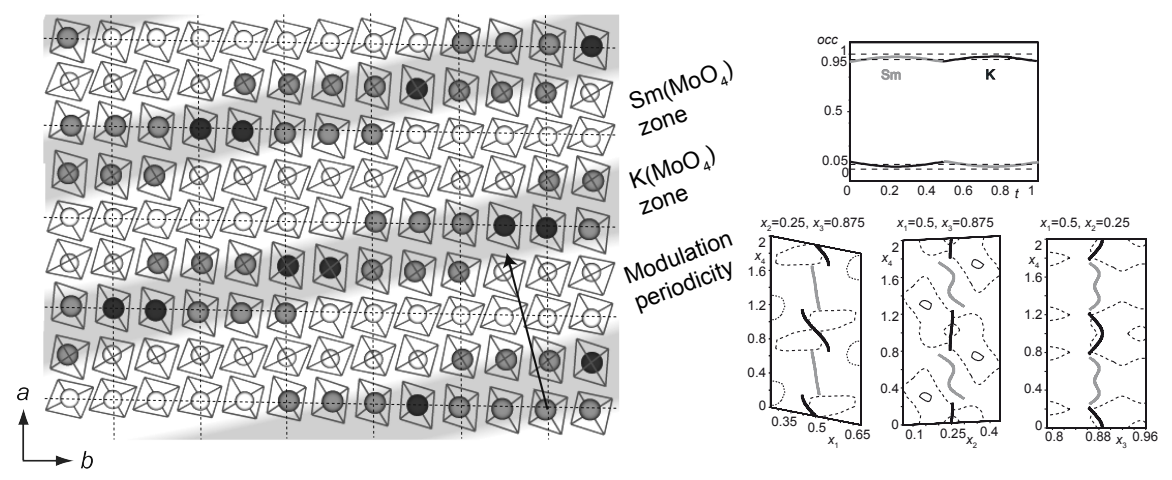

Fig. 7 Left: ab projection of a portion of the aperiodic structure $\mathrm{KSm}\left(\mathrm{MoO}_{4}\right)_{2}$ resulting from the structure refinement. Large circles represent the $\mathrm{K}$ and $\mathrm{Sm}$ whereas the edges represent the $\mathrm{MoO}_{4}$ tetrahedra. Right: 2-d sections resulting from the refinement of the incommensurate structure in superspace. The curves characterize the positions of the Sm and $\mathrm{K}$ atoms and their occupations.

The second example concerns the incommensurate structure $\mathrm{KSm}\left(\mathrm{MoO}_{4}\right)_{2}$ based on synchrotron powder diffraction measurements [18]. Although the ratio $\mathrm{K} / \mathrm{Sm}$ is purely stoichiometric, the resulting structure represented on the left of fig. 7 is remarkable. We observe slanted zones (relative to the lattice vectors) of $\mathrm{Sm}$ and $\mathrm{MoO}_{4}$ alternating with zones of $\mathrm{K}$ and $\mathrm{MoO}_{4}$ normal to the direction of the modulation vector. In 3-d, the structure consists of alternating $\mathrm{K}$ respectively $\mathrm{Sm}$ slabs, the origin of which is not yet explained. This structure results from the $(3+1)$ d refinement illustrated on the right of fig. 7 where three $\left(\mathbf{a}_{S i}, \mathbf{a}_{S 4}\right)$ sections of the $\mathrm{K}$ and Sm positions and their occupation are illustrated. The shape of the curves are obtained by successive approximations and the specialists dispose of a selection of them (see e.g. fig. 4) to apply in their refinements. It is worth mentioning that the model obtained by powder refinement fits perfectly well with the corresponding HREM images.

Up to this point, we have only presented the geometrical characteristics of aperiodic structures. The interesting aspects of structural investigations only start once their resolutions are completed. In the following section, we shall describe an interesting application of the superspace formalism relating structural and physical properties.

\section{Application}

The series of compounds $\mathrm{Na}_{x} \mathrm{Eu}_{(2-x) / 3}^{3+} \mathrm{MoO}_{4}(0 \leqslant x \leqslant 1 / 2)$ belong to the same structural type as presented in section 3 . Eu containing compounds exhibit interesting luminescent properties and one of the main task of material scientists is to optimize these properties according to some adjustable parameters like composition or other. For many years, scientists have vainly attempted to correlate the luminescence efficiency, in particular by the $\mathrm{Na} / \mathrm{Eu}^{3+}$ content. 


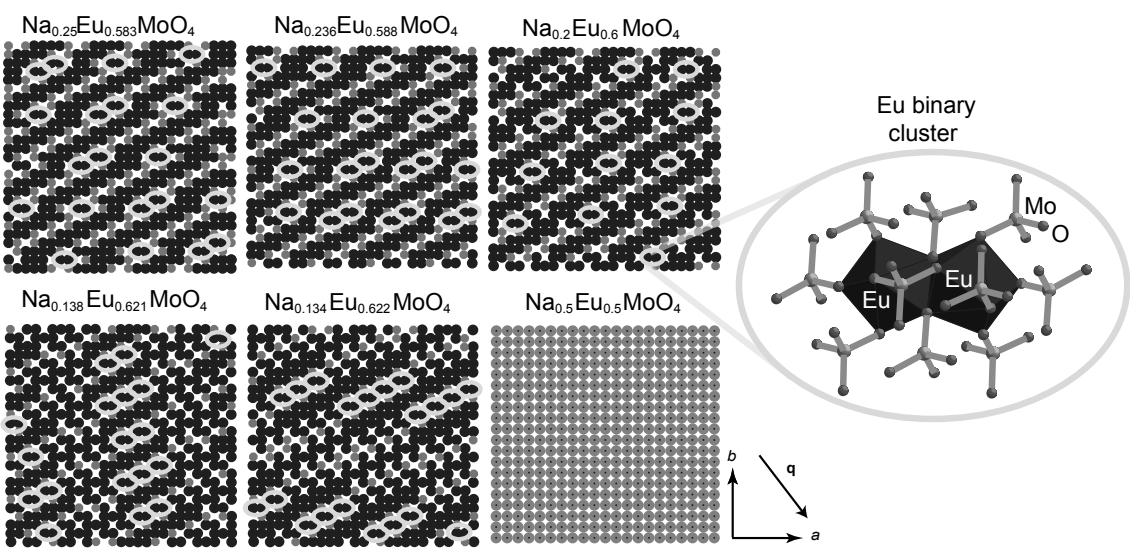

Fig. 8 Schematic $a b$ projections of various incommensurate structures. Only Eu (black circles) and $\mathrm{Na}$ (grey circles) are represented along with vacancies (white stars). Eu dimers are indicated by ellipses. The last structure is fully disordered.

Having recently found that these types of compounds, i.e. scheelites, often form aperiodic structures, we have prepared a series of eight compounds in the given range in order to find a direct correlation between the luminescence characteristics and the structural properties [19]. Six out of eight members of the series exhibit incommensurate structures which have been solved in the superspace formalism. A schematic representation of six structures is given in fig. 8 in the form of 2-d sections. Here again we observe alternating domains of $\mathrm{Na}$ and $\mathrm{Eu}$ rich slabs roughly parallel to the diagonal of the basic cells. In addition we also observe isolated clusters of Eu-pairs, the number of which is independent of the $\mathrm{Na} / \mathrm{Eu}^{3+}$ ratio. It turns out the luminescence efficiencies are directly related to the number of Eu binary clusters as can be seen from fig 9. This interesting result explains why previous attempt to correlate the structures and properties failed because the identification of binary clusters can only be found by solving the structures in superspace (and not by using of a 3-d approximation as is often the case). The second interesting point relates to the optimization of the luminescence (or any other physical) properties by material engineering. Once the superspace symmetry characteristics are identified, it is an easy task to explore the superspace and simulate the best composition which maximize e.g. the number of clusters.

\section{Conclusion}

The discovery of aperiodic structures has changed our vision of the crystalline state and has revealed a more complex organization of matter with long range order different from 3-d periodicity. Thus since more than four decades a new paradigm of structural characteristics had to be introduced in order to ex- 

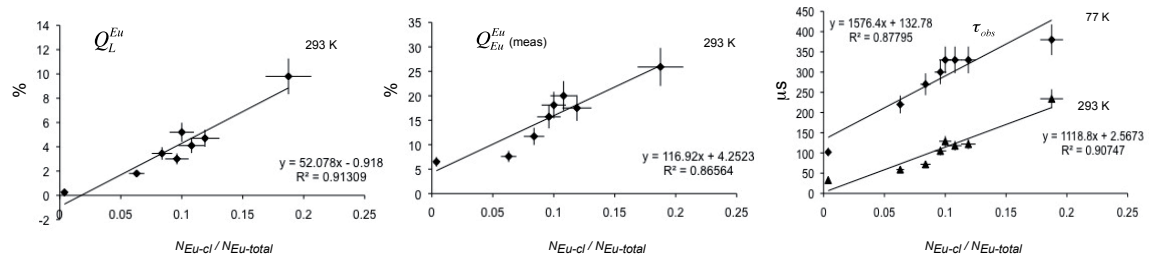

Fig. 9 Various indicators for luminescence efficiencies (quantum yield, lifetimes and intrinsic quantum yield) in relation to the relative amount of $\mathrm{Eu}^{3+}$ dimers for the series of aperiodic structures $\mathrm{Na}_{x} \mathrm{Eu}_{(2-x) / 3}^{3+} \mathrm{MoO}_{4}$.

tend the classical view of 3-d periodic crystals. Every year sees an increasing number of aperiodic structures in every kind of material. The introduction of the superspace formalism has greatly contributed to the rapid evolution of the field and many new possible applications are introduced. In this limited space, only a single example of superspace applications has been presented. Many more have been proposed in the specialized literature. See for example [20-23].

The role of aperiodic structures in structural sciences can no longer be ignored. The availability of synchrotrons and up-to-date detectors has changed the scope of the field. Superspace is a powerful tool not only for the description of aperiodic structures but also for conventional structures and families of structures with common features. It closes the gap between periodic and aperiodic structures. One should also bear in mind that structures which were considered as disordered in the past appears very often to be fully ordered but aperiodic. In other words, they possess long range order There is thus a great potential for superspace in material research sciences for a better understanding of structure-property relations.

Since the discovery of diffraction hundred years ago, our perception of the state of crystalline matter has greatly evolved. The discovery of aperiodic structures has probably done most to the evolution of this perception. The basic tools for their studies being in place, we shall certainly see in the future an increased number of developments which will contribute to the resolution of structural problems not accessible with classical methods.

\section{References}

1. U. Dehlinger, Zeitschrift für Kristallographie 65(5/6), 615 (1927)

2. G. Preston, Proceedings of The Royal Society of London Series A-Mathematical And Physical Sciences 167(A931), 0526 (1938)

3. A. Kochendörfer, Zeitschrift für Kristallographie 101(1/2), 149 (1939)

4. V. Daniel, H. Lipson, Proceedings of the Royal Society of London Series A-Mathematical and Physical Sciences 181(A987), 0368 (1943)

5. M.E. Hargreaves, Acta Crystallographica 4(4), 301 (1951)

6. S. Chao, W. Taylor, Proceedings of the Royal Society of London Series A-Mathematical and Physical Sciences 174(A956), 0057 (1940). DOI 10.1098/rspa.1940.0005 
7. M. Korekawa, Theorie der satellitenreflexe. Habilitationsschrift, Ludwigs-MaximiliansUniversität zu München (1967)

8. P.M.D. de Wolff, W. van Aalst, Acta Crystallographica Section A 28(S), S111 (1972)

9. A. Janner, Acta Crystallographica Section A 28(S), S111 (1972)

10. E. Makovicky, B. Hyde, in Inorganic Chemistry, Structure $\&$ Bonding, vol. 46 (Springer Berlin / Heidelberg, 1981), pp. 101-170

11. D. Shechtman, I. Blech, D. Gratias, J.W. Cahn, Phys. Rev. Lett. 53, 1951 (1984)

12. D. Levine, P.J. Steinhardt, Phys. Rev. Lett. 53, 2477 (1984). DOI 10.1103/PhysRevLett.53.2477. URL http://link.aps.org/doi/10.1103/PhysRevLett.53.2477

13. T. Janssen, G. Chapuis, M. de Boissieu, Aperiodic Crystals: From Modulated Phases to Quasicrystals. International Union of Crystallography Monographs on Crystallography (Oxford University Press, 2007). URL http://books.google.ch/books?id=sHnJ2wBdcjYC

14. S. van Smaalen, Incommensurate Crystallography. International Union of Crystallography Monographs on Crystallography (OUP Oxford, 2007). URL http://books.google.ch/books?id=JOn7OYV7U4UC

15. H.T. Stokes, B.J. Campbell, S. van Smaalen, Acta Crystallographica Section A 67(1), 45 (2011). DOI 10.1107/S0108767310042297. URL http://dx.doi.org/10.1107/S0108767310042297

16. A. Yamamoto, Acta Crystallographica Section A 52(4), 509 (1996). DOI 10.1107/S0108767396000967. URL http://dx.doi.org/10.1107/S0108767396000967

17. W. van Aalst, J. den Holander, W.J.A.M. Peterse, P.M. de Wolff, Acta Crystallographica Section B 32(1), 47 (1976). DOI 10.1107/S056774087600229X. URL http://dx.doi.org/10.1107/S056774087600229X

18. A. Arakcheeva, P. Pattison, G. Chapuis, M. Rossell, A. Filaretov, V. Morozov, G. Van Tendeloo, Acta Crystallographica Section B 64(2), 160 (2008). DOI 10.1107/S0108768108001870. URL http://dx.doi.org/10.1107/S0108768108001870

19. A. Arakcheeva, D. Logvinovich, G. Chapuis, V. Morozov, S.V. Eliseeva, J.C.G. Buenzli, P. Pattison, Chemical Science 3(2), 384 (2012). DOI 10.1039/c1sc00289a

20. A. Arakcheeva, G. Chapuis, Acta Crystallographica Section B 62(1), 52 (2006). DOI 10.1107/S0108768105034671. URL http://dx.doi.org/10.1107/S0108768105034671

21. I. Orlov, L. Palatinus, A. Arakcheeva, G. Chapuis, Acta Crystallographica Section B 63(5), 703 (2007). DOI 10.1107/S0108768107038104. URL http://dx.doi.org/10.1107/S0108768107038104

22. V.A. Morozov, A.V. Arakcheeva, V.V. Konovalova, P. Pattison, G. Chapuis, O.I. Lebedev, V.V. Fomichev, G. Van Tendeloo, Journal of Solid State Chemistry 183(2), 408 (2010). DOI 10.1016/j.jssc.2009.12.008. URL http://www.elsevier.com/locate/jssc

23. L. Elcoro, O. Pérez, J.M. Perez-Mato, V. Petř́iček, Acta Crystallographica Section B 68(4), 341 (2012). DOI 10.1107/S0108768112018095. URL http://dx.doi.org/10.1107/S0108768112018095 